\title{
Indices and regularizations of measurable functions
}

\author{
A. KAMIŃSKA* Department of Mathematical Sciences, The University of Memphis, \\ Memphis, TN 38152, USA \\ L. MALIGRANDA ${ }^{\dagger}$, L. E. PERSSON Department of Mathematics, Luleå University of \\ Technology, S-97187 Luleå, Sweden
}

In the first part we discuss, complete and extend some results on regularizations of measurable functions and their connections to Matuszewska-Orlicz indices. In the second part we prove a certain result on regularization of Young functions and present some applications to study isometries in Calderón-Lozanovskii spaces.

We assume that all (extended) real-valued functions appearing in this paper are Lebesgue measurable and not identically equal to zero. We agree also on the convention $0 \cdot \infty=0$ and that the terms increasing or decreasing will mean non-decreasing or non-increasing, respectively. As usual let $\mathbb{N}, \mathbb{Z}, \mathbb{R}$ and $\mathbb{R}_{+}$stand for the sets of natural numbers, integers, reals and nonnegative reals, respectively. Given $\varphi: \mathbb{R}_{+} \rightarrow \mathbb{R}_{+}$we define the lower and upper (Matuszewska-Orlicz) indices

$$
\begin{aligned}
& \alpha(\varphi)=\sup \left\{p \in \mathbb{R}: \varphi(\lambda u) \leq C \lambda^{p} \varphi(u) \text { for some } C>0 \text { and all } u \geq 0,0<\lambda \leq 1\right\}, \\
& \beta(\varphi)=\inf \left\{p \in \mathbb{R}: \varphi(\lambda u) \leq C \lambda^{p} \varphi(u) \text { for some } C>0 \text { and all } u \geq 0, \lambda \geq 1\right\},
\end{aligned}
$$

respectively ([18], [21], [22], [26]). We say that $\varphi: \mathbb{R}_{+} \rightarrow \mathbb{R}_{+}$and $\psi: \mathbb{R}_{+} \rightarrow$ $\mathbb{R}_{+}$are weakly equivalent if there exist positive constants $C_{1}, C_{2}$ and $K_{1}, K_{2}$ such that $C_{1} \varphi\left(K_{1} u\right) \leq \psi(u) \leq C_{2} \varphi\left(K_{2} u\right)$ for every $u \geq 0$. The functions are said to be equivalent if the previous inequalities are satisfied with $K_{1}=K_{2}=1$. Recall also that $\varphi$ satisfies condition $\Delta_{2}$, if $\varphi(2 u) \leq K \varphi(u)$ for all $u \geq 0$ and some $K>0$. It is easy to show that the $\Delta_{2}$-condition as well as indices are preserved under (weak) equivalence relation between functions. A function $\varphi: \mathbb{R}_{+} \rightarrow \mathbb{R}_{+}$is called pseudo-increasing (resp. pseudo-decreasing) if there exists a constant $K>0$ such that $\varphi(u) \leq K \varphi(v)$ (resp. $\varphi(u) \geq K \varphi(v))$ for any $0 \leq u<v$. It is clear that $0 \leq \alpha(\varphi) \leq \beta(\varphi) \leq \infty$ (resp. $-\infty \leq \alpha(\varphi) \leq \beta(\varphi) \leq 0)$ if $\varphi: \mathbb{R}_{+} \rightarrow \mathbb{R}_{+}$is pseudo-increasing (resp. pseudodecreasing) as well as $1 \leq \alpha(\varphi) \leq \beta(\varphi) \leq \infty$ (resp. $0 \leq \alpha(\varphi) \leq \beta(\varphi) \leq 1$ ) whenever $\varphi(0)=0$ and $\varphi$ is convex (resp. concave).

\footnotetext{
${ }^{*}$ Research supported by a grant from the Swedish Natural Science Research Council (Ö-AH/KG 08685-314)

${ }^{\dagger}$ Research supported by a grant from the Swedish Natural Science Research Council (M-AA/MA 06857-307)
} 


\section{REGULARIZATIONS OF MEASURABLE FUNCTIONS}

Given a measurable function $\varphi: \mathbb{R}_{+} \rightarrow \mathbb{R}_{+}$let's introduce the following cxtended real valued functions defined for all $u>0$,

$$
\begin{aligned}
\Phi(u) & =\int_{0}^{u} \frac{\varphi(t)}{t} d t, \\
\Phi^{0}(u) & =\int_{0}^{u} \sup _{0<s \leq t} \frac{\varphi(s)}{s} d t, \\
\Phi_{0}(u) & =\int_{0}^{u} \inf _{0<s \leq t} \frac{\varphi(s)}{s} d t, \\
\Psi(u) & =u \int_{u}^{\infty} \frac{\varphi(t)}{t^{2}} d t, \\
\Gamma(u) & =u \int_{0}^{u} \frac{\varphi(t)}{t^{2}} d t .
\end{aligned}
$$

Obviously $\Phi_{0} \leq \Phi \leq \Gamma$ and $\Phi \leq \Phi^{0}$. It is also clear that $\Phi, \Phi^{0}, \Phi_{0}, \Gamma$ are increasing, and $\Phi^{0}$ is convex and $\Phi_{0}$ is concave. If in addition $\varphi$ is increasing then $\Psi$ is increasing, $\varphi(u / 2) \leq \Gamma(u), 1 / 2 \varphi(u / 2) \leq \Phi(u), u>0$, and moreover $\Psi$ is concave and $\Gamma$ is convex. We will only show that $\Psi$ is concave that is $\Psi\left(\alpha u_{1}+\beta u_{2}\right) \geq \alpha \Psi\left(u_{1}\right)+$ $\beta \Psi\left(u_{2}\right)$ for every $0<u_{1}<u_{2}$, and $0 \leq \alpha \leq 1, \beta=1-\alpha$, which is equivalent to show that

$$
\beta u_{2} \int_{\alpha u_{1}+\beta u_{2}}^{u_{2}} \frac{\varphi(t)}{t^{2}} d t \geq \alpha u_{1} \int_{u_{1}}^{\alpha u_{1}+\beta u_{2}} \frac{\varphi(t)}{t^{2}} d t .
$$

In fact, in view of monotonicity of $\varphi$,

$$
\begin{aligned}
\beta u_{2} \int_{\alpha u_{1}+\beta u_{2}}^{u_{2}} \varphi(t) \frac{d t}{t^{2}} & \geq \beta u_{2} \varphi\left(\alpha u_{1}+\beta u_{2}\right)\left(\frac{1}{\alpha u_{1}+\beta u_{2}}-\frac{1}{u_{2}}\right) \\
& =\alpha u_{1} \varphi\left(\alpha u_{1}+\beta u_{2}\right)\left(\frac{1}{u_{1}}-\frac{1}{\alpha u_{1}+\beta u_{2}}\right) \\
& \geq \alpha u_{1} \int_{u_{1}}^{\alpha u_{1}+\beta u_{2}} \varphi(t) \frac{d t}{t^{2}} .
\end{aligned}
$$

Convexity of $\Gamma$ may be proved analogously.

The functions listed above are regularizations of measurable nonnegative rcal-valued functions and can be considered as elementary ones. By their different modifications or combinations we obtain new ones. Some possible modifications used in the literature arc the following

$$
\begin{aligned}
\Phi_{1}(u) & =\int_{0}^{u} \inf _{s \geq t>0} \frac{\varphi(s)}{s} d t . \\
\Phi^{1}(u) & =\int_{0}^{u} \sup _{s \geq t>0} \frac{\varphi(s)}{s} d t, \\
\Psi^{0}(u) & =u \int_{u}^{\infty} \sup _{0<s \leq t} \frac{\varphi(s)}{s^{2}} d t, \quad(u>0)
\end{aligned}
$$


and analogously $\Psi_{0}, \Psi_{1}, \Psi^{1}, \Gamma^{0}, \Gamma_{0}, \Gamma_{1}, \Gamma^{1}$.

There are numerous examples of applications of either the listed regularizations themselves or their modifications or their combinations. At the end of this section we will refer to some of them in geometry of classical function (Banach or linear metric) spaces and in interpolation of operators in these spaces. Frequently in applications it is important to find regularizations that bear certain required properties and such that they are (weakly) equivalent to a given function $\varphi$. In the propositions below we list in a systematic way many properties of $\Phi, \Phi^{0}, \Phi_{0}, \Psi$ and $\Gamma$, we link them to indices and we give necessary and sufficient conditions on when they are (weakly) equivalent to $\varphi$. Although most of these results are well known or may be found partially in different sources (e.g. [5], [15], [18], [21]-[27], [29]), they often appear in not complete versions. We will provide here the proofs of the first two propositions, concavity related, and skip the analogous proofs of their convexity counterparts.

PROPOSITION 1.1 Let $\varphi: \mathbb{R}_{+} \rightarrow \mathbb{R}_{+}$be such that $\varphi(u) / u$ is pseudo-decreasing and $\Phi(u)$ is finite for every $u>0$. Then the following conditions are equivalent.

(1) $\Phi$ and $\varphi$ are equivalent.

(2) $\Phi$ and $\varphi$ are weakly equivalent.

(3) $\Phi_{0}$ and $\varphi$ are equivalent.

(4) $\Phi_{0}$ and $\varphi$ are weakly equivalent.

(5) $\alpha(\varphi)>0$.

(6) $\alpha(\Phi)>0$.

(7) $\alpha(\Phi)=\alpha(\varphi)>0$.

(8) For any $\lambda>1$

$$
\inf _{u>0} \Phi(\lambda u) / \Phi(u)>1
$$

(9) For some $\lambda>1$

$$
\inf _{u>0} \Phi(\lambda u) / \Phi(u)>1
$$

Proof: In order to see that (1) and (3) are equivalent and (2) and (4) as well, it is enough to observe that for all $u>0$ and some $K>0$,

$$
K \varphi(u) / u \leq \inf _{0<s \leq u} \varphi(s) / s \leq \varphi(u) / u
$$

and so

$$
K \Phi(u) \leq \Phi_{0}(u) \leq \Phi(u) .
$$

(5) $\Rightarrow$ (6). The assumption $\alpha(\varphi)>0$ implies that $\varphi(\lambda u) \leq C \lambda^{p} \varphi(u)$ for some $C>0, p>0$ and all $u \geq 0$. Hence for $\lambda \geq 1$,

$$
\Phi(\lambda u)=\int_{0}^{\lambda u} \frac{\varphi(t)}{t} d t=\int_{0}^{u} \frac{\varphi(\lambda t)}{\lambda t} \lambda d t \leq C \lambda^{p} \int_{0}^{u} \frac{\varphi(t)}{t} d t=C \lambda^{p} \Phi(u),
$$

showing that $\alpha(\Phi)>0$.

The implication (6) $\Rightarrow(9)$ is a direct consequence of the definition of indices.

(9) $\Rightarrow(1)$. By the assumption that $\varphi(u) / u$ is pseudo-decreasing,

$$
\Phi(u)=\int_{0}^{u} \frac{\varphi(t)}{t} d t \geq K \varphi(u) .
$$


Moreover, if $D>1$ is such that $\Phi(\lambda u) \geq D \Phi(u)$ for all $u>0$, then

$$
K^{-1}(\lambda-1) \varphi(u) \geq \int_{u}^{\lambda u} \frac{\varphi(t)}{t} d t=\Phi(\lambda u)-\Phi(u) \geq(D-1) \Phi(u) .
$$

which implies that $\varphi(u) \geq C \Phi(u)$ for all $u>0$ and some $C>0$. Thus $\varphi$ and $\Phi$ arc equivalent.

$(2) \Rightarrow(8)$. Since $\varphi$ is weakly equivalent to $\Phi, \Phi(u) \leq C_{2} \varphi\left(K_{2} u\right), C_{2}, K_{2}>0$ Moreover for any $\lambda>1$,

$$
\Phi(\lambda u)-\Phi(u)=\int_{u}^{\lambda u} \frac{\varphi(t)}{t} d t \geq K \frac{\varphi(\lambda u)}{\lambda u}(\lambda-1) u=C \varphi(\lambda u)
$$

for some $C>0$. Hence

$$
\Phi(\lambda u) / \Phi(u)-1 \geq C \varphi(\lambda u) / \Phi(u) \geq C C_{2}^{-1} \Phi\left(\left(\lambda / K_{2}\right) u\right) / \Phi(u) .
$$

Observe also that for any $0<\beta<1$,

$$
\Phi(\beta u)=\int_{0}^{\beta u} \frac{\varphi(t)}{t} d t=\int_{0}^{u} \frac{\varphi(\beta t)}{\beta t} \beta d t \geq K \int_{0}^{u} \frac{\varphi(t)}{t} \beta d t=K \beta \Phi(u) .
$$

Thus

$$
\Phi\left(\left(\lambda / K_{2}\right) u\right) \geq K \min \left(\lambda / K_{2}, 1\right) \Phi(u),
$$

and so for certain $M>0$ and all $u>0$

$$
\Phi(\lambda u) / \Phi(u) \geq M+1>1 .
$$

$(9) \Rightarrow(6)$. Without loss of generality we can assume that condition (9) is true for $\lambda=2$. Letting $D=\inf _{u>0} \Phi(2 u) / \Phi(u)>1$, for any $u>0, \Phi(u / 2) \leq D^{-1} \Phi(u)$. For $0<\lambda \leq 1$, there exists a nonnegative integer $n$ such that $1 / 2^{n+1}<\lambda \leq 1 / 2^{n}$. Setting $q=\ln (1 / D) / \ln (1 / 2), q>0$ and $1 / D^{n}=\left(1 / 2^{n}\right)^{q}$, we find that

$$
\Phi(\lambda u) \leq \Phi\left(\left(1 / 2^{n}\right) u\right) \leq\left(1 / D^{n}\right) \Phi(u)=\left(1 / 2^{n}\right)^{q} \Phi(u) \leq 2^{q} \lambda^{q} \Phi(u),
$$

which shows that $\alpha(\Phi)>0$.

$(6) \Rightarrow(7)$. Since we have already showed that (6) is equivalent to (1), $\Phi$ and $\varphi$ are equivalent, which implies that $\alpha(\varphi)=\alpha(\Phi)>0$.

Thus we finished the proof since the other implications are obvious.

REMARKS 1. Each of the conditions (1)-(9) yields that $\varphi$ is pseudo-increasing. In fact, $\varphi$ considered in Proposition 1.1 is pseudo-concave, i.e. for some $C>0$ and all $s, t>0$. $\varphi(s) \leq C \max (1, s / t) \varphi(t)$. Any increasing subadditive function $\varphi: \mathbb{R}_{+} \rightarrow \mathbb{R}_{+}$is an example of pseudo-concave function, since $\varphi(s) \leq 2 \max (1, s / t) \varphi(t)$ for all $s, t>0$. Notice also that if $\alpha(\varphi)>0$ then $\Phi$ is finite.

2. The assumption in Proposition 1.1 that $\Phi$ must be finite is essential, since there exists an increasing function $\varphi$ such that $\varphi(u) / u$ is decreasing and yet $\Phi(u)=\infty, u>0$. It is enough to define $\varphi(u)=1 /|\ln u|$ for $u \in\left(0, e^{-1}\right)$ and then extend it appropriately on the whole $\mathbb{R}_{+}$.

3. The converse of Proposition 1.1 does not hold in the sense that there exists an increasing function $\varphi$ such that $\varphi(u) / u$ is decreasing, $\Phi$ is finite on the whole $\mathbb{R}_{+}$and yet 
$\alpha(\varphi)=0$. Given $1<p<\infty$, define $\varphi(u)=1 /(|\ln u|)^{p}$ around zero and extend it to infinity in such a manner that it satisfies the required properties. We easily check that $\varphi(u) / u$ is decreasing and that $\Phi$ is finite. We also get for any $q>0$ that

$$
\lim _{u \rightarrow 0} \varphi(u) / u^{q}=\infty,
$$

which yields that the lower index of $\varphi$ is zero. By Proposition 1.1, $\varphi$ can not be weakly equivalent to $\Phi$. In fact there do not exist constants $D$ and $C$ such that $\Phi(t) \leq C \varphi(D t)$, since the inequality $(1 / 2) \varphi(u) \leq \Phi(u)$ is always true by concavity of $\Phi$. Thus even if the function $\Phi$ is well defined it does not need to be (weakly) equivalent to $\varphi$.

PROPOSITION 1.2 Let $\varphi: \mathbb{R}_{+} \rightarrow \mathbb{R}_{+}$be pseudo-increasing and $\varphi(u) / u$ be pseudodecreasing. Assume also that $\Psi(u)$ is finite for every $u>0$. Then the following conditions are equivalent.

(1) $\Psi$ and $\varphi$ are equivalent.

(2) $\Psi$ and $\varphi$ are weakly equivalent.

(3) $\beta(\varphi)<1$.

(4) $\beta(\Psi)<1$.

(5) $\beta(\varphi)=\beta(\Psi)<1$.

(6) For any $\lambda>1$

$$
\sup _{u>0} \Psi(\lambda u) / \lambda \Psi(u)<1 .
$$

(7) For some $\lambda>1$

$$
\sup _{u>0} \Psi(\lambda u) / \lambda \Psi(u)<1 .
$$

Proof: In order to complete the proof it is enough to show the implications below.

(3) $\Rightarrow$ (1) If $\beta(\varphi)<1$, then $\varphi(u) / u^{1-\epsilon}$ is pseudo-decreasing for some $\epsilon>0$. Hence for some $K>0$ and all $u>0$,

$$
\Psi(u) \leq K u \frac{\varphi(u)}{u^{1-\epsilon}} \int_{u}^{\infty} \frac{d t}{t^{1+\epsilon}}=(K / \epsilon) \varphi(u) .
$$

Moreover, for any $u>0$ and some $C>0$,

$$
\Psi(u) \geq u \int_{u}^{2 u} \frac{\varphi(t)}{t} \frac{d t}{t} \geq(C / 2) \varphi(2 u) \int_{u}^{2 u} \frac{d t}{t} \geq((C / 2) \ln 2) \varphi(u),
$$

that shows the equivalence of $\varphi$ and $\Psi$.

(2) $\Rightarrow(6)$ Denote $S(u)=\int_{u}^{\infty} w(t) / t d t$, where $w(t)=\varphi(t) / t$. By the assumptions that $w$ is pseudo-decreasing and that $\varphi$ is weakly equivalent to $\Psi$, there exists $K>0$, such that for all $u>0, S(u) \leq K w(u)$. Thus for any $\lambda>1$ and $u>0$,

$$
\begin{aligned}
\Psi(\lambda u) / \lambda \Psi(u) & =S(\lambda u) / S(u)=1-\int_{u}^{\lambda u} w(t) \frac{d t}{t} / S(u) \\
& \leq 1-K^{-1} w(\lambda u) \int_{u}^{\lambda u} \frac{d t}{t} / K w(u) \leq 1-(\ln \lambda) w(\lambda u) / K^{2} w(u) \\
& =1-(\ln \lambda) \varphi(\lambda u) / K^{2} \lambda \varphi(u) \leq 1-M,
\end{aligned}
$$

where $M>0$ is a constant dependent only on $\lambda$. 
(7) $\Rightarrow$ (1) We have

$$
\Psi(u) \geq u \int_{u}^{2 u} \frac{\varphi(t)}{t} \frac{d t}{t} \geq u(C \ln 2) \frac{\varphi(2 u)}{2 u} \geq K \varphi(u)
$$

for all $u>0$ and some $K>0$. On the other hand letting

$$
A=\sup _{u>0} \Psi(\lambda u) / \lambda \Psi(u)
$$

$A<1$ and so for $u>0$

$$
0<1-A \leq 1-S(\lambda u) / S(u)=\int_{u}^{\lambda u} w(t) \frac{d t}{t} / S(u) \leq C w(u) \ln \lambda / S(u) .
$$

which implies that

$$
\Psi(u)=u S(u) \leq M \varphi(u),
$$

for all $u>0$ and some $M>0$.

(7) $\Rightarrow$ (4) By the assumption, there exists $A$ such that $\lambda A>1$ and $\Psi(\lambda u) \leq \lambda A \Psi(u)$ for all $u>0$. Now let $q=\ln (\lambda A) / \ln \lambda$ and $\beta \geq 1$ be arbitrary. Thus there exists an integer $n$ such that $\lambda^{n} \leq \beta \leq \lambda^{n+1}$ and so for some $K>0$,

$$
\begin{aligned}
\Psi(\beta u) & \leq K \Psi\left(\lambda^{n+1} u\right) \leq K(\lambda A)^{n+1} \Psi(u)=K\left(\lambda^{n+1}\right)^{q} \Psi(u) \\
& =K\left(\lambda^{n}\right)^{q} \lambda^{q} \Psi(u) \leq K \beta^{q} \lambda^{q} \Psi(u) .
\end{aligned}
$$

which shows that $\beta(\Psi)<1$. We used here the fact that $\Psi$ is pseudo-increasing.

(4) $\Rightarrow$ (7) Since $\beta(\Psi)<1$ there exist $0<\epsilon<1$ and $K>0$ such that for all $\lambda>1$ and $u>0$,

$$
\frac{\Psi(\lambda u)}{\lambda \Psi(u)}=\frac{1}{\lambda^{\epsilon}}\left(\frac{\Psi(\lambda u)}{(\lambda u)^{I-\epsilon}}\right) /\left(\frac{\Psi(u)}{u^{1-\epsilon}}\right) \leq \frac{K}{\lambda^{\epsilon}},
$$

which easily shows (7) since $\lim _{\lambda \rightarrow \infty} K / \lambda^{\epsilon}=0$.

$(4) \Rightarrow(3)$ and $(4) \Rightarrow(5)$ We have already showed that (4) is equivalent to (7) and that (7) implies (1). Thus $\varphi$ must be equivalent to $\Psi$. Now (3) and (5) follow instantly.

PROPOSITION 1.3 If $\varphi: \mathbb{R}_{+} \rightarrow \mathbb{R}_{+}$is such that $\varphi(u) / u$ is pseudo-increasing, then the functions $\varphi$ and $\Phi$ (resp. $\varphi$ and $\Phi^{0}$ ) are weakly equivalent, that is

$$
K^{-1} \varphi((1 / 2) u) \leq \Phi(u) \leq K \varphi(u) \quad\left(\text { resp. } \quad \varphi((1 / 2) u) \leq \Phi^{0}(u) \leq K \varphi(u)\right)
$$

for some $K>0$ and all $u>0$.

PROPOSITION 1.4 Let $\varphi: \mathbb{R}_{+} \rightarrow \mathbb{R}_{+}$be such that $\varphi(u) / u$ is pseudo-increasing. The following conditions are equivalent.

(1) $\Phi$ and $\varphi$ are equivalent.

(2) $\Phi^{0}$ and $\varphi$ are equivalent.

(3) $\varphi$ satisfies condition $\Delta_{2}$.

(4) $\Phi$ satisfies condition $\Delta_{2}$.

(5) $\Phi^{0}$ satisfies condition $\Delta_{2}$. 
(6) $\beta(\Phi)<\infty$.

(7) $\beta\left(\Phi^{0}\right)<\infty$.

(8) $\beta(\varphi)<\infty$.

(9) $\beta(\varphi)=\beta\left(\Phi^{0}\right)=\beta(\Phi)<\infty$.

(10) For all $\lambda>1$

$$
\sup _{u>0} \varphi(\lambda u) / \varphi(u)<\infty
$$

(11) For some $\lambda>1$

$$
\sup _{u>0} \varphi(\lambda u) / \varphi(u)<\infty
$$

PROPOSITION 1.5 Let $\varphi: \mathbb{R}_{+} \rightarrow \mathbb{R}_{+}$be such that $\varphi(u) / u$ is pseudo-increasing. Assume also that $\Gamma(u)$ is finite for every $u \geq 0$. Consider the following conditions.

(1) $\Gamma$ and $\varphi$ are equivalent.

(2) $\Gamma$ and $\varphi$ are weakly equivalent.

(3) $\alpha(\varphi)>1$.

(4) $\alpha(\Gamma)>1$.

(5) $\alpha(\varphi)=\alpha(\Gamma)>1$.

(6) For any $\lambda>1$

$$
\inf _{u>0} \Gamma(\lambda u) / \lambda \Gamma(u)>1
$$

(7) For some $\lambda>1$

$$
\inf _{u>0} \Gamma(\lambda u) / \lambda \Gamma(u)>1 .
$$

Then the conditions (3), (4), (5) and (7) are equivalent and (3) implies (4) and (1) implies (6). If in addition $\varphi$ satisfies $\Delta_{2}$, then all above conditions are equivalent.

Condition $\Delta_{2}$ is a necessary assumption in the above proposition in order to get all conditions equivalent. In fact, if we consider the function $\varphi(u)=e^{u^{2}-1}$, then $\alpha(\varphi)=2$ and $\beta(\varphi)=\infty$, and $\varphi$ is not equivalent to $\Gamma$.

The next two theorems are improvements of the similar results contained in [5], [21][27] and [29]. The methods of their proofs are based on regularizations discussed in the previous propositions.

THEOREM 1.6 Let $0 \leq a<b<\infty$. If $\varphi: \mathbb{R}_{+} \rightarrow \mathbb{R}_{+}$is such that $u^{-a} \varphi(u)$ is pseudoincreasing and $u^{-b} \varphi(u)$ is pseudo-decreasing, then there exists an increasing function $\psi$ equivalent to $\varphi$ such that $\psi\left(u^{1 / b}\right)$ is concave. If in addition $a>0$, then $\psi\left(u^{1 / a}\right)$ is also convex.

Proof: By the assumption there exists $C>0$ such that

$$
\varphi(s) \leq C \max \left(s^{a} / t^{a}, s^{b} / t^{b}\right) \varphi(t)
$$

for all $s, t>0$. Setting

$$
\varphi_{0}(t)=t^{a} \sup _{s>0} \min \left(s^{-a}, t^{b-a} s^{-b}\right) \varphi(s),
$$

$\varphi(t) \leq \varphi_{0}(t) \leq C \varphi(t)$ and $t^{-a} \varphi_{0}(t)$ is increasing and $t^{-b} \varphi_{0}(t)$ is decreasing. 
If $a>0$ then define

$$
\psi(u)=\int_{0}^{u} \frac{\varphi_{0}(t)}{t} d t
$$

Since $b^{-1} \varphi_{0}(t) \leq \psi(t) \leq a^{-1} \varphi_{0}(t), \psi$ is equivalent to $\varphi$ and $\psi\left(u^{1 / a}\right)=a^{-1} \int_{0}^{\prime \prime} \varphi_{0}\left(t^{1 / a}\right) / t d t$ is convex and $\psi\left(u^{1 / h}\right)=b^{-1} \int_{0}^{u} \varphi_{0}\left(t^{1 / h}\right) / t d t$ is concave.

For $a=0, \varphi_{1}(u)=\varphi_{0}\left(u^{1 / b}\right)$ is increasing and $\varphi_{1}(u) / u$ is decreasing. Thus there exists a concave majorant $\tilde{\varphi}_{1}$ equivalent to $\varphi_{1}$ (cf. Remark 6 or just take $\tilde{\varphi}_{1}(u)=\inf _{t>0}(1+$ $u / t) \varphi(t), u>0)$, and setting $\psi(u)=\tilde{\varphi}_{1}\left(u^{b}\right)$ we obtain the required function.

THEOREM 1.7 Let $0 \leq a<b<\infty$ and $\varphi: \mathbb{R}_{+} \rightarrow \mathbb{R}_{+}$be pscudo-increasing. If $a<\alpha(\varphi) \leq \beta(\varphi)<b$, then there exists an increasing function $\psi \in C^{1}(0 . \infty)$ equivalent to $\varphi$ and such that for some $\epsilon>0$ the functions $\psi\left(u^{1 /(u+\epsilon)}\right)$ and $\psi\left(u^{1 /(b-\epsilon)}\right)$ are convex and concave, respectively. In particular $\psi(u) / u^{a+\epsilon}$ and $\psi(u) / u^{b-\epsilon}$ are increasing and decreasing, respectively. If in addition $\varphi$ is continuous, then $\psi \in C^{2}(0, \infty)$.

Proof: Define

$$
\psi_{0}(u)=\Gamma_{a}(u)+\Psi_{b}(u)
$$

where

$$
\Gamma_{a}(u)=u^{a} \int_{0}^{u} s^{-u} \varphi(s) \frac{d s}{s} \text { and } \Psi_{b}(u)=u^{b} \int_{u}^{\infty} s^{-b} \varphi(s) \frac{d s}{s} .
$$

Since $\varphi(t) / t^{a+\epsilon}$ is pseudo-increasing for some $\epsilon>0$.

$$
\int_{0}^{u} s^{-u} \varphi(s) \frac{d s}{s} \leq K \frac{\varphi(u)}{u^{a+\epsilon}} \int_{0}^{u} \frac{d s}{s^{1-\epsilon}}<\infty,
$$

which yiclds that $\Gamma_{a}$ is finite. Analogously we show that $\Psi_{b}$ is finitc.

Both functions $\Gamma_{a}$ and $\Psi_{b}$ are equivalent to $\varphi$. In fact, if $a=0$ then $\Gamma_{a}=\Phi$ and then we apply Proposition 1.1. If $a>0$ then $\Gamma_{a}\left(u^{1 / a}\right)=a^{-1} u \int_{0}^{u} \varphi\left(s^{1 / a}\right) / s^{2} d s$, which is the function $\Gamma$ computed for $\varphi\left(t^{1 / a}\right)$. The latter function satisfies condition $\Delta_{2}$ in view of the assumption that $\beta(\varphi)<\infty$ and also $\varphi\left(u^{1 / a}\right) / u$ is pscudo-increasing by $\alpha(\varphi)>a$. Applying now Proposition 1.5 to the function $\varphi\left(u^{1 / a}\right)$ we obtain that it is equivalent to $\Gamma_{a}\left(u^{1 / a}\right)$ or that $\varphi$ is equivalent to $\Gamma_{a}$. Analogously we apply Proposition 1.2 to the function $\varphi\left(u^{1 / b}\right)$ in order to show that $\Psi_{b}$ is equivalent to $\varphi$. Computing derivatives of $\psi_{0}(u) / u^{a+\epsilon}$ and $\psi_{0}(u) / u^{b-\epsilon}$ we get that they are increasing and decreasing, respectively. Finally, letting

$$
\psi(u)=\int_{0}^{u} \frac{\psi_{0}(t)}{t} d t .
$$

we finish the proof since $\psi$ is equivalent to $\psi_{0}$ and hence to $\varphi$, and moreover $\psi\left(u^{1 /(u+\epsilon)}\right)$ and $\psi\left(u^{1 /(h-\epsilon)}\right)$ are convex and concave, respectively.

REMARKS 4. In the theory of Orlicz spaces the function $\Phi^{0}$ was used by Mazur and Orlicz [27] (see also [22], [25]) in order to show that the Orlicz space $L_{\varphi}$ gencrated by an increasing function $\varphi$ is locally convex iff $\varphi$ is equivalent to a convex function. The 
regularizations presented in Propositions 1.1, 1.3, 1.4 and their various modifications, are frequently used in the theory of Orlicz, Lorentz and Musielak-Orlicz spaces. Maleev and Troyanski [20] and Figiel [4] as well as Altshuler [1] have applied them to estimate the modulus of convexity in Orlicz spaces and Lorentz spaces, respectively. In order to study type and cotype and also order convexities and concavities in these spaces, the regularizations appear also a very useful method (cf. [9], [10], [13], [18]). Observe also that particularly in Orlicz type spaces, many modified regularizations are employed, and they in general depend on the measure space ([21]-[26]). For instance, if the measure space is finite and nonatomic then only the behaviour of $\varphi$ for large arguments is essential, and then all conditions are assumed only at infinity. In the sequence case the values of $\varphi$ are essential only for small arguments. Also in these spaces usually weak equivalence is enough for applications (cf. [14], [18], [21], [22]).

5. Theorems 1.6 and 1.7 have some applications in the interpolation theory. Theorem 1.6, in case of $a=0$ and $b=1$, have been used by Peetre (cf. [2], Th. 5.4.4) in order to show that $h: \mathbb{R}_{+} \rightarrow \mathbb{R}_{+}$is an interpolation function of power $p, 0<p \leq \infty$, (i.e. if any linear operator $T$ which is continuous from $L^{p}\left(u_{0}\right)$ to $L^{p}\left(v_{1}\right)$ is also continuous from $L^{p}\left(u_{0} h\left(u_{1} / u_{0}\right)\right)$ to $L^{p}\left(v_{0} h\left(v_{1} / v_{0}\right)\right)$ and $M \leq C \max \left(M_{0}, M_{1}\right)$ for some constant $C$, where the $M^{\prime} s$ are appropriate norms of $T$ ) if and only if $h$ is pseudo-concave.

Theorem 1.7, again for $a=0$ and $b=1$, is applied e.g. in the proof of the reiteration theorem for the $K$-method of interpolation with a function parameter

$$
\left(\left(A_{0}, A_{1}\right)_{\varphi_{0}, q_{0}},\left(A_{0}, A_{1}\right)_{\varphi_{1}, q_{1}}\right)_{\varphi_{2}, q}=\left(A_{0}, A_{1}\right)_{\varphi_{0} \varphi_{2}\left(\varphi_{1} / \varphi_{0}\right), q},
$$

where $0<\alpha\left(\varphi_{i}\right) \leq \beta\left(\varphi_{i}\right)<1: i=0,1,2$ and either $0<\alpha\left(\varphi_{1} / \varphi_{0}\right) \leq \beta\left(\varphi_{1} / \varphi_{0}\right)<\infty$ or $0<\alpha\left(\varphi_{0} / \varphi_{1}\right) \leq \beta\left(\varphi_{0} / \varphi_{1}\right)<\infty$ (see [5], Th. 2.3 and [29], Prop. 4.3). The restrictions on indices correspond to the usual condition $\theta_{0} \neq \theta_{1}$ in the power case (see [2], Th. 3.5.3).

6. It is worth to notice that by Propositions 1.1 and 1.3 , if $\varphi: \mathbb{R}_{+} \rightarrow \mathbb{R}_{+}$is increasing and $\varphi(u) / u$ is decreasing (resp. $\varphi(u) / u$ is increasing), then $\Phi(u)$ is a concave majorant of $\varphi$ equivalent to $\varphi$ whenever $\alpha(\varphi)>0$ (resp. convex minorant weakly equivalent to $\varphi$ ). But in general they are not the least concave majorant or the greatest convex minorant, respectively. However the latter functions exist whenever $\varphi(0)=0, \varphi$ is increasing and $\varphi(u) / u$ is decreasing or increasing respectively. In fact, already in 1961, Stechkin (see [3], Lemma 4) introduced the least concave majorant by the formula

$$
\begin{aligned}
\psi(u) & =\sup \left\{\frac{u_{2}-u}{u_{2}-u_{1}} \varphi\left(u_{1}\right)+\frac{u-u_{1}}{u_{2}-u_{1}} \varphi\left(u_{2}\right): 0 \leq u_{1} \leq u<u_{2}\right\} \\
& =\sup \left\{\alpha \varphi\left(u_{1}\right)+(1-\alpha) \varphi\left(u_{2}\right): 0 \leq \alpha \leq 1, u_{1}, u_{2} \geq 0, \alpha u_{1}+(1-\alpha) u_{2}=u\right\} .
\end{aligned}
$$

In 1970, Peetre [28] gave the following formula of the least concave majorant

$$
\psi(u)=\sup \left\{\sum_{i=1}^{n} \lambda_{i} \varphi\left(u_{i}\right): u=\sum_{i=1}^{n} \lambda_{i} u_{i}, u_{i} \geq 0, \sum_{i=1}^{n} \lambda_{i}=1, \lambda_{i} \geq 0, n \in \mathbb{N}\right\}
$$

and in 1978, Lozanovskii [19] introduced another one

$$
\psi(u)=\inf _{s>0} \sup _{v>0} \frac{s+u}{s+v} \varphi(v) .
$$

Switching in the Stechkin's or Peetre's formula from supremum to infimum, we obtain the convex greatest minorant weakly equivalent to $\varphi$ whenever $\varphi(u) / u$ is increasing (cf. [30]). We should finally mention here that the convex greatest minorant is also given by $\varphi^{* *}$ where $\varphi^{*}(u)=\sup _{v>0}(u v-\varphi(v))$ is a Legendre transformation of $\varphi$ ([7]). 


\section{ISOMETRIES IN CALDERÓN-LOZANOVSKII SPACES}

In this section we will prove a certain regularization result for Young functions and then we will apply it to discuss isometries in Caledrón-Lozanovskii spaces.

A function $\varphi: \mathbb{R}_{+} \rightarrow \mathbb{R}_{+}$is said to be a Young function whenever it is convex, vanishes only at zero and $\varphi(1)=1$. We will use further many properties of $\varphi$ that are satisfied only for large arguments. By adding the term "for 1.a." after a property we will indicate that it holds for all arguments that are larger than a certain number. For instance we say that $\varphi$ satisfies condition $\Delta_{2}$ for l.a., if there exist $C>0, u_{0} \geq 0$ such that the inequality $\varphi(2 u) \leq C \varphi(u)$ is fulfilled for all $u \geq u_{0}$. In case of lower and upper indices of $\varphi$, if they are defined by inequalities satisfied only for large arguments, then we denote them by $\alpha^{\infty}(\varphi)$ and $\beta^{\infty}(\varphi)$, respectively (cf. [21]). It is also easy to show that they are preserved under weak equivalence relation for large arguments between Young functions.

THEOREM 2.1 Let $\varphi$ and $\psi$ be Young functions such that $\psi$ satisfies condition $\Delta_{2}$ for l.a. and their quotient $\varphi(u) / \psi(u)$ is pseudo-decreasing for l.a.. Then there exists a Young function $\gamma$ equivalent for l.a. to $\varphi$, such that $\gamma \circ \psi^{-1}$ is concave.

Proof: In view of the assumption that the quotient is pseudo-decreasing for I.a. and that $\psi$ satisfies condition $\Delta_{2}$ for l.a., there exist $\mu_{0} \geq 0$ and $K, C>1$ such that

$$
\varphi(2 u) \leq K \frac{\psi(2 u)}{\psi(u)} \varphi(u) \leq K C \varphi(u),
$$

for $u \geq u_{0}$. Thus $\varphi$ also satisfies condition $\Delta_{2}$ for l.a.. Recall also that for any Young function $\chi$ satisfying $\Delta_{2}$-condition for l.a. with the constants $A>1$ and $u_{0} \geq 0$ it holds

$$
A^{-2} u \chi^{\prime}(u) \leq A^{-2} \chi(2 u) \leq \chi(u / 2) \leq(u / 2) \chi^{\prime}(u / 2) \leq \chi(u) \leq u \chi^{\prime}(u) .
$$

for $u \geq u_{0}$, where $\chi^{\prime}$ is the left-derivative of $\chi$ ([14]). Definc

$$
r(u)= \begin{cases}\varphi^{\prime}\left(u_{0}\right) / \psi^{\prime}\left(u_{0}\right), & \text { for } 0 \leq u \leq u_{0} \\ \inf _{u_{0} \leq t \leq u} \varphi^{\prime}(t) / \psi^{\prime}(t), & \text { for } u>u_{0} .\end{cases}
$$

Applying now the $\Delta_{2}$-condition of $\varphi$ and $\psi$, for all $u_{0} \leq t \leq u$.

$$
\varphi^{\prime}(t) / \psi^{\prime}(t) \geq C^{-2} \varphi(t) / \psi(t) \geq K^{-1} C^{-2} \varphi(u) / \psi(u),
$$

which yields that for all $u \geq u_{0}$,

$$
C^{-1} K^{-2} \varphi(u) / \psi(u) \leq r(u) \leq \varphi^{\prime}(u) / \psi^{\prime}(u) .
$$

Define

$$
\gamma(u)=\int_{0}^{u} r(t) \psi^{\prime}(t) d t, \quad(u \geq 0) .
$$

By the above inequalities, for all $u \geq 2 u_{0}$,

$$
\begin{aligned}
\gamma(u) & \leq \int_{0}^{\left.u_{0}\right)} \frac{\varphi^{\prime}\left(u_{0}\right)}{\psi^{\prime}\left(u_{0}\right)} \psi^{\prime}(t) d t+\int_{u_{0}}^{u} \frac{\varphi^{\prime}(t)}{\psi^{\prime}(t)} \psi^{\prime}(t) d t \leq \varphi^{\prime}\left(u_{0}\right) u_{0}+\varphi(u) \\
& \leq(K C)^{2} \varphi\left(u_{0}\right)+\varphi(u) \leq\left((K C)^{2}+1\right) \varphi(u),
\end{aligned}
$$


and

$$
\gamma(u) \geq \int_{u_{0}}^{u} r(t) \psi^{\prime}(t) d t \geq r(u) \int_{u / 2}^{u} \psi^{\prime}(t) d t \geq \frac{1}{2} r(u) \psi(u) \geq\left(C^{-1} K^{-2} / 2\right) \varphi(u),
$$

which show that $\gamma$ and $\varphi$ are equivalent for 1.a.. In order to show that $\gamma$ is convex we will prove that the function $r(u) \psi^{\prime}(u)$ is increasing. Given $u_{0} \leq u_{1} \leq u_{2}$ the following inequalities are satisfied

$$
\begin{gathered}
r\left(u_{1}\right) \psi^{\prime}\left(u_{1}\right)=\psi^{\prime}\left(u_{1}\right) \inf _{u_{0} \leq t \leq u_{1}} \varphi^{\prime}(t) / \psi^{\prime}(t) \leq \psi^{\prime}\left(u_{2}\right) \inf _{u_{0} \leq t \leq u_{1}} \varphi^{\prime}(t) / \psi^{\prime}(t), \\
r\left(u_{1}\right) \psi^{\prime}\left(u_{1}\right)=\psi^{\prime}\left(u_{1}\right) \inf _{u_{0} \leq t \leq u_{1}} \varphi^{\prime}(t) / \psi^{\prime}(t) \leq \varphi^{\prime}\left(u_{1}\right)=\psi^{\prime}\left(u_{2}\right) \inf _{u_{1} \leq t \leq u_{2}} \varphi^{\prime}\left(u_{1}\right) / \psi^{\prime}(t) \\
\leq \psi^{\prime}\left(u_{2}\right) \inf _{u_{1} \leq t \leq u_{2}} \varphi^{\prime}(t) / \psi^{\prime}(t) .
\end{gathered}
$$

Hence

$$
r\left(u_{1}\right) \psi^{\prime}\left(u_{1}\right) \leq \psi^{\prime}\left(u_{2}\right) \min \left\{\inf _{u_{0} \leq t \leq u_{1}} \phi^{\prime}(t) / \psi^{\prime}(t), \inf _{u_{1} \leq t \leq u_{2}} \varphi^{\prime}(t) / \psi^{\prime}(t)\right\} \leq \psi^{\prime}\left(u_{2}\right) r\left(u_{2}\right),
$$

which shows that $r(u) \psi^{\prime}(u)$ is increasing. Finally, changing variables,

$$
\gamma\left(\psi^{-1}(u)\right)=\int_{0}^{u} r\left(\psi^{-1}(t)\right) d t,
$$

which yields concavity of $\gamma \circ \psi^{-1}$, since $r \circ \psi^{-1}$ is decreasing.

We also observe that we can replace $r(u)$ equivalently by $p(u)$, where

$$
p(u)= \begin{cases}\varphi\left(u_{0}\right) / u_{0} \psi^{\prime}\left(u_{0}\right), & \text { for } 0 \leq u \leq u_{0} \\ \inf _{u_{0} \leq t \leq u} \varphi(t) / t \psi^{\prime}(t), & \text { for } u>u_{0}\end{cases}
$$

COROLLARY 2.2 Let $\chi$ be a Young function such that $1<\alpha^{\infty}(\chi) \leq \beta^{\infty}(\chi)<\infty$. Then there exist Young functions $\varphi$ and $\psi$ such that $\psi \circ \varphi=\chi$ and none of them is weakly equivalent for l.a. to any power function.

Proof: Let's choose a Young function $\gamma$ such that $\alpha^{\infty}(\chi)>\beta^{\infty}(\gamma)>\alpha^{\infty}(\gamma) \geq 1$ and $\beta^{\infty}(\chi) / \beta^{\infty}(\gamma) \neq \alpha^{\infty}(\chi) / \alpha^{\infty}(\gamma)$. For any $\beta^{\infty}(\gamma)<p<\alpha^{\infty}(\chi)$, the functions $\chi(u) / u^{p}$ and $\gamma(u) / u^{p}$ are pseudo-increasing and pseudo-decreasing for l.a., respectively. Hence for some $u_{0} \geq 0$ and $K>0$ and all $u_{0} \leq u<v$,

$$
\frac{\gamma(u)}{\chi(u)}=\frac{\gamma(u)}{u^{p}} / \frac{\chi(u)}{u^{p}} \geq K \frac{\gamma(v)}{v^{p}} / K^{-1} \frac{\chi(v)}{v^{p}}=K^{2} \frac{\gamma(v)}{\chi(v)},
$$

that is $\gamma / \chi$ is pseudo-decreasing for l.a.. By the previous result, there exists a Young function $\varphi$ equivalent for la. to $\gamma$ and such that $\varphi \circ \chi^{-1}$ is concave. Letting $\psi=\chi \circ \varphi^{-1}$, $\psi$ is convex and $\psi \circ \varphi=\chi$.

The function $\varphi$ is not weakly equivalent for l.a. to any power function since $\alpha^{\infty}(\varphi)=$ $\alpha^{\infty}(\gamma)<\beta^{\infty}(\gamma)=\beta^{\infty}(\varphi)$. Now, if we assume that $\psi$ is weakly equivalent for la. to a power function $u^{p}$ for some $1 \leq p<\infty$, then $\varphi^{-1}(u)$ is weakly equivalent for 1.a. to $\chi^{-1}\left(u^{p}\right)$. This yields by properties of indices that

$$
\beta^{\infty}(\chi) / \beta^{\infty}(\varphi)=\beta^{\infty}(\chi) / \beta^{\infty}(\gamma)=p=\alpha^{\infty}(\chi) / \alpha^{\infty}(\gamma)=\alpha^{\infty}(\chi) / \alpha^{\infty}(\varphi),
$$

and we obtain a contradiction which completes the proof. 
By $L^{0}$ we denote the space of all Lebesgue measurable real or complex valued functions on $[0,1]$. Let further $\left(E,\|\|_{E}\right.$ ) (a subspace of $L^{0}$ ) be a rearrangement invariant function space (for definition see [18], p.117), which will be called shortly an r.i. space. We also assume here that $\left\|\mathbf{1}_{[0,1]}\right\|_{E}=1$ and that $\left(E,\|\|_{E}\right)$ satisfies the Fatou property ([15], [18]).

Given a r.i. space $E$ and a Young function $\varphi$, the Calderón-Lozanovskii space $E_{\varphi}$ is then the set of all functions $f \in L^{0}$ such that $\|f\|<\infty$, where

$$
\|f\|=\inf \{a>0: \rho(f / a) \leq 1\},
$$

and $\rho(f)=\|\varphi(|f|)\|_{E}$ if $\varphi(|f|) \in E$ and $\rho(f)=\infty$ otherwise. It is well known that ( $\left.E_{\varphi},\|\|\right)$ is also a ri. space [6]. If $E=L^{1}$ then $E_{\varphi}$ is an Orlicz space $L_{\varphi}$ equipped with the Luxemburg norm \|\|$_{\varphi}([14])$ and if $\varphi(u)=u^{p} .1 \leq p<\infty$, then $E_{\varphi}=E^{(p)}$ is a $p$-convexification of $E$ endowed with the norm $\|f\|_{(p)}=\left\|f^{p}\right\|_{E}^{1 / p}$ ([18]). Observe also that if $\varphi$ and $\psi$ are Young functions and if $E=L_{\psi}$, then $E_{\varphi}=\left(L_{\psi}\right)_{\varphi}=L_{\psi \circ \varphi}$.

Given a Banach space $\left(X,\|\|_{X}\right)$, by an isometry of $X$ we mean further a linear surjective operator of $X$, and we denote the group of all isometries of $X$ by Iso $(X)$. We say that two Banach spaces are equal up to equivalence of norm (resp. isometrically), if they coincide as sets and the norms are equivalent (resp. the norms arc equal).

For any measurable subset $A \subset[0,1]$, let $|A|$ denote its Lebcsgue measure. An invertible Borel measurable function $\sigma:[0,1] \rightarrow[0,1]$ such that $\left|\sigma^{-1}(A)\right|=0$ iff $|A|=0$ for any Borel set $A \subset[0,1]$ will be called an automorphism of $[0,1]$. The set of all automorphisms of $[0,1]$ will be denoted by $N S$. An automorphism $\sigma$ is called measure preserving if for any Borel set $A \subset[0,1],\left|\sigma^{-1}(A)\right|=|A|$. Given $\sigma, \sigma^{\prime}$ denotes the Radon-Nikodym derivative $d \mu \circ \sigma^{-1} / d \mu$ and clcarly $\sigma^{\prime}(t)=1$ a.e. on $[0,1]$ for a measure preserving automorphism. An operator $U$ on $L^{0}$ is called an elementary operator if there exist $\sigma \in N S$ and $h \in L^{0}$ such that $U f=h f \circ \sigma$. An elementary operator with $|h|=1$ is an isometry on a r.i. space $E$.

Given a r.i. space $E$, denote by $p(E)$ and $q(E)$ the Boyd indices of $E$ ([18]). Recall the following results on isometries of r.i. spaces.

\section{THEOREM A}

I. ([8], [34]) If $E$ is a r.i. space not equal isometrically to $L^{2}$, then any isometry of $E$ is an elementary operator.

II. ([8]) If $E$ is a r.i. space and $U$ is an isometry of $E$, then either $E=L^{p}$ up to equivalence of norm for some $1 \leq p \leq \infty$ or there exist a measure preserving automorphism $\sigma$ of $[0,1]$ and $h \in L^{0}$ with $|h|=1$ a.e. such that $U f=h f \circ \sigma$ for all $f \in E$.

If additionally $E$ is not equal isometrically to $L^{2}$, then $U$ is also an isometry of $L^{r}$ for any $p(E) \leq r \leq q(E)$.

For any ri. space $E$ there exists a 1-1 correspondence between all positive isometrics of $E$ and a subgroup of $N S$. By this correspondence we identify the group Iso $(E)$ with an associated subgroup of $N S$. Thus we shortly write that Iso $\left(L^{p}\right)=N S, 1 \leq p \leq$ $\infty, p \neq 2$, since it is wcll known, that for any $\sigma \in N S$ there exists $h \in L^{0}$ such that $U f=h(f \circ \sigma)$ is an isometry of $L^{p}$. Moreover, if $1 \leq p<\infty, p \neq 2$, then $|h|^{p}=\sigma^{\prime}$ a.e., and if $p=\infty$ then $|h|=1$ a.e. ([32]). The groups of isometries of ri. spaces have been classified in [17] and [34]. 
PROPOSITION 2.3 If $E$ is a r.i. space such that Iso $(E)$ coincides with $N S$, then there exists $1 \leq p \leq \infty, p \neq 2$, such that $E=L^{p}$ isometrically.

Proof: By Theorem A, $E=L^{p}$ for some $1 \leq p \leq \infty$ and \|\|$_{E}$ is equivalent to \|\|$_{p}$.

Let $p=\infty$. For any $\sigma \in N S$, there exists $h \in L^{0}$ such that $U f=h f \circ \sigma$ is an isometry of $\left(E,\|\|_{E}\right)$. Since $p(E)=q(E)=\infty$, by Theorem A, $U$ is also an isometry of $L^{\infty}$, and hence $|h|=1$. Thus for any $B \subset[0,1],\left\|\mathbf{1}_{B}\right\|_{E}=\left\|U \mathbf{1}_{B}\right\|_{E}=\left\|h \mathbf{1}_{\sigma^{-1}(B)}\right\|_{E}=$ $\left\|\mathbf{1}_{\sigma^{-1}(B)}\right\|_{E}$. Then in view of the Fatou property of $E,\left\|\mathbf{1}_{B}\right\|_{E}=\left\|\mathbf{1}_{[0,1]}\right\|_{E}=1$, which yields that the norms in $E$ and $L^{\infty}$ are identical.

Now let $1 \leq p<\infty, p \neq 2$. Then we have the equality Iso $(E)=$ Iso $\left(L^{p}\right)$. Indeed, by Theorem A, since the both Boyd indices of $E$ and $L^{p}$ are equal to $p$, any isometry $U$ of $E$ is also an isometry of $L^{p}$, whence there exist $\sigma \in N S$ and $h \in L^{0}$, such that $U f=h f \circ \sigma$ and $\sigma^{\prime}=|h|^{p}$ a.e.. Since any $\sigma \in N S$ generates an isometry in $E$ as well as in $L^{p}$, Iso $(E)=$ Iso $\left(L^{p}\right)$.

Now take an arbitray function $f$ in $E$ with $\|f\|_{p}=1$ and let $\|f\|_{E}=r$. By almost transitivity of \|\|$_{p}([31])$,

$$
\begin{aligned}
S_{p}(1) & =\left\{g:\|g\|_{p}=1\right\}=\overline{\left\{U f: U \in \operatorname{Iso}\left(L^{p}\right)\right\}} \\
& =\overline{\{U f: U \in \operatorname{Iso}(E)\}} \subset\left\{g:\|g\|_{E}=r\right\}=S_{E}(r) .
\end{aligned}
$$

Hence $S_{p}(1)=S_{E}(r)$, and since $\left\|\mathbf{1}_{[0,1]}\right\|_{E}=\left\|\mathbf{1}_{[0,1]}\right\|_{p}=1$, so $r=1$. Therefore $\|f\|_{E}=\|f\|_{p}$.

In the case when $E$ is an Orlicz space $L_{\varphi}$, the classification of the group of all isometries depends on the group of multipliers of $\varphi$. Recall that $b>0$ is a multiplier of a Young function $\varphi$ if $\varphi(b u)=\varphi(b) \varphi(u)$ for all $u \geq 0$. Denoting by $M_{\varphi}$ the group of multipliers of $\varphi$, it is well known that either $M_{\varphi}=\mathbb{R}_{+}$and then $\varphi$ is a power function, or $M_{\varphi}$ is a cyclic group generated by $a>1$ or $M_{\varphi}=\{1\}$ ([16]). The following theorem classifies the group of isometries in Orlicz spaces.

THEOREM B ([33]) The group of all isometries of an Orlicz space $L_{\varphi}$ coincides with one of the following subgroups.

(1) $N S$, when $\varphi(u)=u^{p}, 1 \leq p<\infty, p \neq 2$.

(2) $N S\left(a^{p} ; 1\right)=\left\{\sigma \in N S: \sigma^{\prime}(t) \in\left\{\left(a^{p}\right)^{k}\right\}_{k=-\infty}^{\infty}\right.$ a.e. on $\left.[0,1]\right\}$, when $\varphi$ is equivalent to $u^{p}, 1 \leq p<\infty, p \neq 2$, and $M_{\varphi}$ is a cyclic group generated by $a>1$.

(3) $P=\{\sigma \in N S: \sigma$ is measure preserving $\}$ in all other cases.

The goal of classifying isometries of Calderón-Lozanovskii spaces is rather a complex problem. Some information may be obtained by estimating the Boyd indices of $E_{\varphi}$. Let $F$ denote the fundamental function of $E$, that is $F(u)=\left\|1_{[0, u]}\right\|_{E}$ and let $\tilde{F}(u)=1 / F(1 / u), u \in(0,1)$.

PROPOSITION 2.4 If $\alpha\left(\tilde{F}^{-1} \circ \varphi\right)<\beta\left(\tilde{F}^{-1} \circ \varphi\right)$, then Iso $\left(E_{\varphi}\right)=P$.

If $p(E)=\infty$ then either Iso $\left(E_{\varphi}\right)=P$ or $E=L^{\infty}$.

If $\alpha\left(\tilde{F}^{-1} \circ \varphi\right)=1$ then either Iso $\left(E_{\varphi}\right)=P$ or $E=L^{1}$ and $\varphi(u)=u$ for all $u \in \mathbb{R}_{+}$.

Proof: In view of Theorem 5 in [12], $p\left(E_{\varphi}\right) \leq \alpha\left(\tilde{F}^{-1} \circ \varphi\right)<\beta\left(\tilde{F}^{-1} \circ \varphi\right) \leq q\left(E_{\varphi}\right)$, and so Iso $\left(E_{\varphi}\right)=P$ by Theorem $\mathrm{A}$.

If $p(E)=\infty$, then again by Theorem 5 in [12], $\infty=p(E) \alpha^{\infty}(\varphi) \leq p\left(E_{\varphi}\right)$, which yields that either Iso $\left(E_{\varphi}\right)=P$ or $E_{\varphi}=L^{\infty}$ isometrically ([17], [34]). The latter equality 
clearly implies that $E_{\varphi}=L^{\infty}$ as sets. Moreover, for any $f$ with $\|f\|_{\infty}=1$ we have $\left\|\varphi^{-1}(|f|)\right\|_{\infty}=1$ and so $\left\|\varphi^{-1}(|f|)\right\|=1$. Therefore $\|f\|_{E}=\left\|\varphi\left(\varphi^{-1}(|f|)\right)\right\|_{E}=1$. Hence $\|f\|_{\infty}=\|f\|_{E}$ for any $f \in E$.

If $\alpha\left(\tilde{F}^{-1} \circ \varphi\right)=1$ then $p\left(E_{\varphi}\right)=1$ and by Theorem 1 in [17], cither Iso $\left(E_{\varphi}\right)=P$ or $E_{\varphi}=L^{1}$ isometrically. By order continuity of the norm in $E_{\varphi}, \varphi$ satisfies condition $\Delta_{2}$ (Corollary 5 in [6]). Thus for any $f \in E_{\varphi}$ with $\|f\|=1$ it holds $\rho(f)=1$. Therefore if $\|f\|=\|f\|_{1}$, then $\rho\left(f /\|f\|_{1}\right)=\left\|\varphi\left(|f| /\|f\|_{1}\right)\right\|_{E}=1$. Hence for any $0<a<1$, letting $f=(1 / a) \mathbf{1}_{[0 . a]}$, we have $\|f\|_{1}=1$ and

$$
1=\left\|\varphi\left((1 / a) \mathbf{1}_{[0, a]}\right)\right\|_{E} \geq(1 / a)\left\|\mathbf{1}_{[0, a]}\right\|_{E} .
$$

On the other hand, since $\left\|\left(\int_{0}^{1} f\right) 1_{[0.1]}\right\|_{E} \leq\|f\|_{E}$ for any $f \in E$. we get the reverse inequality

$$
a \leq\left\|\mathbf{1}_{[0, a]}\right\|_{E}
$$

Thus $\left\|\mathbf{1}_{[0, a]}\right\|_{E}=a$ for any $0<a<1$, and so $\|f\|_{E}=\|f\|_{1}$ for any $f \in E$. Moreover, for any $0<a<1$,

$$
1=\left\|\varphi\left((1 / a) \mathbf{1}_{[0 . a]}\right)\right\|_{E}=\varphi(1 / a)\left\|\mathbf{1}_{[0, a]}\right\|_{E}=\varphi(1 / a) a,
$$

which means that $\varphi(u)=u$ for all $u \geq 0$ in view of convexity of $\varphi$.

Below we present a variety of possibilities that may occur in the group of isomctries of $E_{\varphi}$ under different choices of $E$ and $\varphi$. Basically there are not direct connections among properties of $\varphi$, Iso $(E)$ and Iso $\left(E_{\varphi}\right)$.

(1). For any $1<p<\infty$, there exist a r.i. space $E$ and a Young function $\varphi$ such that Iso

$(E)=P, \varphi$ is not equivalent to a power function, but $E_{\varphi}=L^{p}$. Thus if $p \neq 2$, then Iso $\left(E_{\varphi}\right)=N S$.

Indeed, by Corollary 2, we can find two Young functions $\varphi$ and $\psi$ such that none of them is equivalent to a power function, but $\psi \circ \varphi(u)=u^{p}$. Now, if we take $E=L_{\psi}$, then Iso $\left(L_{\psi}\right)=P$ by Theorem B and $E_{\varphi}=\left(L_{\psi}\right)_{\varphi}=L_{\psi \circ \varphi}=L^{p}$.

(2). There exist a r.i. space $E$ with Iso $(E)=P$ and a Young function $\varphi$ not equivalent to any power function such that Iso $\left(E_{\varphi}\right)=N S(a ; 1)$ for some $a>1$.

Let $\chi$ be a Young function with a cyclic group of multipliers; for cxample we can take $\chi(u)=u^{p} \exp \sin (\ln u), 1<p<\infty$, with a multiplier $a=e^{2 \pi}$ ([16]). Then, by Theorem $\mathrm{B}$, Iso $\left(L_{\chi}\right)=N S(a ; 1)$. By Corollary 2 , one can find two Young functions $\varphi$ and $\psi$ such that $\psi \circ \varphi=\chi$ and none of them is equivalent to a power function. Letling $E=L_{\psi}$. Iso $(E)=P$ and $E_{\varphi}=L_{\psi \circ \varphi}=L_{\chi}$ and so Iso $\left(E_{\varphi}\right)=N S(a ; 1)$.

(3). Let $\varphi$ and $\psi$ be Young functions such that none of $\varphi, \psi$ or $\psi \circ \varphi$ is equivalent to a power function; e.g. $\varphi(u)=u^{p} \ln (u+1), 1<p \neq 2<\infty$ and $\psi(u)=e^{u}-1$. Then setting $E=L_{\psi}$, Iso $\left(E_{\varphi}\right)=$ Iso $E=P$.

(4). Let's assume now that Iso $(E)=N S$. Then, by Proposition 2.3, $E$ is isometrically equal to $L^{p}$ for some $1 \leq p \leq \infty, p \neq 2$. Suppose that $p<\infty$. Then, since $E=L^{p}$, $E_{\varphi}=L_{\varphi}$ p is an Orlicz space. Now it is easy to find three different Young functions $\varphi$ such that the group of isometries Iso $\left(E_{\varphi}\right)$ will coincide with either $N S$ or $N S(a ; 1)$ or $P$. For example let's take as $\varphi$, a power function, a function with a cyclic group of multipliers, and the one not satisfying condition $\Delta_{2}$, respectively.

(5). Let $E=L_{\psi}$, where $\psi(u)=u^{p} \exp \sin (\ln u), 1<p<\infty, p \neq 2$. Then Iso $(E)=N S(a ; 1)$, where $a=e^{2 \pi}$. If $\varphi$ does not have any multipliers, then Iso $\left(E_{\varphi}\right)=P$. 
If $\varphi$ is a power function, then $\psi \circ \varphi$ has a cyclic group of multipliers, and then Iso $\left(E_{\varphi}\right)=$ $N S(a: 1)$. Finally, for $r>p$, let $\varphi(u)=\psi^{-1}\left(u^{r}\right)$. Then $\varphi$ is convex, $\psi \circ \varphi(u)=u^{p}$, and so Iso $\left(E_{\varphi}\right)=N S$.

\section{REFERENCES}

[1] Z. Altshuler, Uniform convexity in Lorentz sequence spaces, Israel J. Math. 20 (1975), 260-274.

[2] .J. Bergh and J. Löfström, Interpolation Spaces. An Introduction, Springer-Verlag, Berlin-Heidelberg-New York, 1976.

[3] A. V. Efimov, Linear methods of approximation of continuous periodic functions, Mat. Sb. 54 (1961), 51-90. (Russian)

[4] T. Figiel, On the moduli of convexity and smoothness, Studia Math. 56 (1976), 121155.

[5] J. Gustavsson, A function parameter in connection with interpolation of Banach spaces, Math. Scand. 42 (1978), 289-305.

[6] H. Hudzik, A. Kamińska and M. Mastyło, Geometric properties of some CalderónLozanovskii spaces and Orlicz-Lorentz spaces, Houston J. Math. 22 (1996), 639-663.

[7] A. D. Ioffe and V. M. Tihomirov, Theory of Extremal Problems, Studies in Math. and its Appl. 6, North-Holland, Amsterdam-New York, 1979.

[8] N. J. Kalton and B. Randrianantoanina, Surjective isometries on rearrangementinvariant spaces, Quart. J. Math. Oxford 45 (1994), 301-327.

[9] A. Kamińska, Indices, convexity and concavity in Musielak-Orlicz spaces, Funct. et Approx. 26 (1998), 67-84.

[10] A. Kamińska and L. Maligranda, Order convexity and concavity in Lorentz spaces with arbitrary weight, to appear.

[11] A. Kamińska, L. Maligranda and L. E. Persson, Type, cotype and convexity properties of Orlicz spaces, Facultad de Matematicas Universidad Complutense, Colloq. Depart. Anal. Mat., Curs 1996-97, No 42, 113-126.

[12] A. Kamińska, L. Maligranda and L. E. Persson, Indices, convexity and concavity of Calderón-Lozanovskii spaces, to appear.

[13] A. Kamińska and B. Turett, Type and cotype in Musielak-Orlicz spaces, Geometry of Banach Spaces, Cambridge University Press, London Math. Soc., Lect. Notes Series 158 (1990), 165-180.

[14] M. A. Krasnoselskii and Ya. B. Rutickii, Convex Functions and Orlicz spaces, Noordhoff Groningen 1961.

[15] S. G. Krein, Ju. I. Petunin and E. M. Semenov, Interpolation of Linear Operators, AMS Translation of Math. Monog. 54, 1982.

[16] J. Lamperti, On the isometries of certain function spaces, Pacific J. Math. 8 (1958), 459-466.

[17] P. K. Lin, Maximality of rearrangement invariant spaces, Bull. Polish Acad. Sciences, 44 (1996), 381-390.

[18] J. Lindenstrauss and L. Tzafriri, Classical Banach Spaces I, II, Springer-Verlag, 1977, 1979.

[19] G. Ya. Lozanovskii, Transformations of ideal Banach spaces by means of concave functions, Qualitative and Approximate Methods for the Investigation of Operator Equations, Yaroslavl, 3 (1978), 122-147 (Russian). 
[20] R. P. Maleev and S. L. Troyanski, On the moduli of convexity and smoothness in Orlicz spaces, Studia Math. 54 (1975), 131-141.

[21] L. Maligranda, Indices and Interpolation, Dissertations Math. 234, Warsaw 1985.

[22] L. Maligranda, Orlicz Spaces and Interpolation, Seminars in Math. 5, Univ. of Campinas, Campinas SP, Brazil 1989.

[23] W. Matuszewska, Some further properties of $\varphi$-functions, Bull. Acad. Polon. Sci.. Ser. Sci. Math. Astronom. Phys. 9 (1961), 445-450.

[24] W. Matuszewska, Regularly increasing functions in connection with the theory of $L^{* \varphi}$ spaces, Studia Math. 21 (1962), 317-344.

[25] W. Matuszewska and W. Orlicz, A note on the theory of $s$-normed spaces of $\varphi$ integrable functions, Studia Math. 21 (1961), 107-115; reprinted in: Władysław Orlicz Collected Papers, PWN, Warszawa 1988, 1148-1156.

[26] W. Matuszewska and W. Orlicz, On certain properties of $\varphi$-functions, Bull. Acad. Polon. Sci., Ser. Sci. Math. Astronom. Phys. 8 (1960), 439-443; reprinted in: Władysław Orlicz Collected Papers, PWN, Warszawa 1988, 1112-1116.

[27] S. Mazur and W. Orlicz, On some classes of linear spaces, Studia Math. 17 (1958), 97-119; reprinted in: Władysław Orlicz Collected Papers, PWN, Warszawa 1988. 981-1003.

[28] J. Peetre, Concave majorants of positive functions, Acta Math. Acad. Scient. Hung. 2 (1970), 327-333.

[29] L. E. Persson, Interpolation with a parameter function, Math. Scand. 59 (1986), 199222.

[30] A. W. Roberts and D. E. Varberg, Convex Functions, Academic Prcss, New York 1973.

[31] S. Rolewicz, Metric Linear Spaces, Polish Scientific Publishers and D. Reidel Publishing Company, Warszawa, Dordrecht / Boston / Lancaster, 1984.

[32] H. L. Royden, Real Analysis, Macmillan Publishing Company and Collier Macmillan Publishing, New York, London, 1988.

[33] M. G. Zaidenberg, Groups of isometries of Orlicz spaces, Sov. Mat. Dokl. 17 (1976), $432-436$.

[34] M. G. Zaidenberg, On isometric classification of symmetric spaces, Sov. Mat. Dokl. 18 (1977), 636-640. 


\section{function spaces}

the fifth conference:

proceedings of

the conference

at Poznań, Poland

edited by

Henryk Hudzik

Leszek Skrzypczak

Adam Mickiewicz University

Poznań, Poland

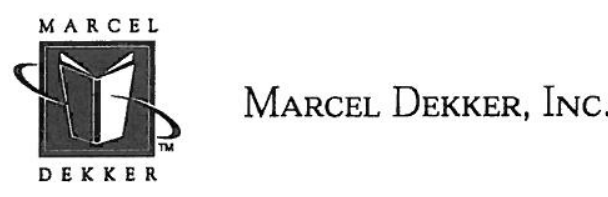

New YoRK · BASEL

Copyright $\odot 2000$ by Marcel Dekker, Inc. All Rights Reserved. 